\title{
La evaluación axiológica de los Proyectos en los Comités de Ética de la investigación
}

\author{
The axiological assessment of projects into research ethics committees
}

\begin{abstract}
Daniel Palma-Morgado' · Roberto Marín-Gil² · Lorena González-García ${ }^{3}$ Jaime Torelló-Iserte ${ }^{4}$ José Manuel Santos-Lozano ${ }^{5} \cdot$ Manuel Ortega-Calvo ${ }^{6}$

1. Farmacéutico de Atención Primaria. Distrito Sanitario de Atención Primaria de Sevilla. Vocal del Comité Coordinador de Ética de la Investigación Biomédica de Andalucía (CCEIBA).

2. Farmacéutico de Hospital. Secretario del Comité de Ética de la Investigación del Hospital Virgen del Rocío de Sevilla.

3. Farmacéutica. Grupo de Investigación en Atención Farmacéutica, Universidad de Granada, Servicio de Farmacia, Hospital Universitario San Cecilio, Granada, España. FIBAO.

4. Farmacólogo Clínico. Centro Andaluz de Fármacovigilancia. Vocal del Comité Coordinador de Ética de la Investigación Biomédica de Andalucía (CCEIBA). Hospital Virgen del Rocío de Sevilla.

5. Médico de Familia. Centro de Salud San Pablo. Profesor Asociado de Medicina de Familia. Departamento de Medicina. Universidad de Sevilla. CIBER de la Fisiopatología de la Obesidad y de la Nutrición. Instituto de Salud Carlos III. Madrid. España.

6. Médico de Familia. Centro de Salud Esperanza Macarena. Vocal del Comité de Ética de la Investigación del Hospital Virgen del Rocío y Virgen Macarena de Sevilla. Unidad de Investigación Distrito Sanitario de Atención Primaria. Sevilla. CIBER de la Fisiopatología de la Obesidad y de la Nutrición. Instituto de Salud Carlos III. Madrid. España.
\end{abstract}

\section{Artículo de Opinión Opinion Article}

\author{
Correspondencia \\ Correspondence \\ Manuel Ortega-Calvo. \\ Av. de la Cruz del Campo 36. Bl. $12^{\circ} \mathrm{A}$. \\ 41005-Sevilla. \\ 106mayorque104@gmail.com \\ Financiación \\ Fundings \\ Este artículo no ha recibido financiación \\ específica \\ Conflicto de interés \\ Competing interest \\ Nno existen conflictos de interese.
}

Received: 01.01.2015 Accepted: 02.02.2015

\section{RESUMEN}

El objetivo de este trabajo es demostrar que la existencia de los Comités de Ética de la Investigación (CEIs) es congruente con el tiempo histórico que nos ha tocado vivir. La idea de una ciencia neutra se remonta a la creación de la Royal Society. Putnam y otros investigadores propusieron el término de valores epistémicos para los valores propios de la ciencia. A esta transformación axiológica se le ha denominado de varias formas: modo 2 de conocimiento, ciencia postnormal, ciencia postacadémica y también tecnociencia. No hay innovación sin valoración previa, e incluso sin múltiples valoraciones previas. La existencia actual de los Comités de Ética de la Investigación (CEIs) es un hecho congruente con el desarrollo histórico de la epistemología durante el siglo XX. Una de las funciones más importantes de los CEIs, es la evaluación axiológica (valores epistémicos) de los proyectos de investigación.

Palabras clave: conocimiento, ética de la investigación, comités de ética, estudios de evaluación.

\begin{abstract}
The aim of this paper is to show that the existence of Ethics Research Committees is consistent with the historical time in which we reside. The idea of a neutral science dates back to the creation of the Royal Society. Putnam and other researchers proposed the term «epistemic values» to the values of science. This axiological transformation has been called in several ways: knowledge mode 2, post-normal science, post-academic science and technoscience. There is no innovation without evaluation, even without multiple previous assessments. The actual existence of the Research Ethics Committees (RECs) is a fact consistent with the historical development of epistemology in the twentieth century. One of the most important functions of RECs, is the axiological evaluation (epistemic values) of research projects.
\end{abstract}

Keywords: knowledge; ethics research; ethics committees; evaluation studies. 
If I have seen further it is by standing on ye shoulders of giants

Sir IsAaC NewTon (1642-1727)

\section{INTRODUCCIÓN}

A finales del siglo XIX y principios del XX, el positivismo afirmaba que el avance científico estaba exento de valores o que éstos no tenían importancia en su desarrollo ${ }^{1}$. La idea de neutralidad en la ciencia se remontaba a la creación de la Royal Society y al documento en el que la institución se comprometía con la monarquía británica, el Royalist Compromise. En él se aseguraba que los científicos tendrían libertad de investigación siempre que no se involucrasen en cuestiones religiosas, políticas o morales ${ }^{2}$.

Augusto Comte (1798-1857) en su Catecismo Positivista alegaba que la ciencia tiene que ver con los hechos pero no con los valores. Investigadores de la talla de Henri Poincaré (1854-1912) y de Albert Einstein (1879-1955) se sumaron a esta corriente en el momento histórico que les tocó vivir. Max Weber (1864-1920) trasladó esa perspectiva a las ciencias sociales al propugnar la «libertad de valores» (Wertfreiheit $)^{3}$. Es decir, los economistas y los sociólogos debían de adoptar una postura neutral cuando investigaran. En la tradición empirista y positivista los juicios de valor eran subjetivos y por lo tanto caían fuera del discurso científico. El primer Wittgenstein ( 1889-1951) mantuvo incluso unas posturas más radicales ${ }^{4}$. En el Tractatus logico-philosophicus (1921) se pueden leer frases como: «En el mundo todo es y sucede como sucede, en él no hay ningún valor...».

En pleno siglo $\mathrm{XX}$ acontece un cambio importante en esta concepción según lo expuesto en la obra «El colapso de la dicotomía hechos/valores» de Hilary Putnam (1926 - ) $)^{5}$. Con posterioridad a la Segunda Guerra Mundial, el desarrollo científico ha tenido más en cuenta los esquemas axiológicos y dentro de estos últimos, los valores. Robert K Merton (1910-2003) afirmó en 1942 que la ciencia posee un ethos ${ }^{6}$ basado en cuatro pilares: comunalismo, universalismo, desinterés y escepticismo. John Ziman (1925-2005) añadió un quinto pilar en el año 2000, la originalidad ${ }^{7}$. Aunque han existido discusiones acerca de este último principio, no debemos olvidar que la ciencia generalmente se construye $« A$ hombros de gigantes» parafraseando al gran Newton. Estos hechos históricos han dado lugar a una disciplina nueva, la ética de la ciencia.

Nuestro objetivo en este artículo es intentar demostrar que la existencia de los Comités de Ética de la Investigación es un hecho coherente con el desarrollo de la epistemología y de la historia de la ciencia en el siglo XX y que además de la pura evaluación ética, en ellos tienen cabida también otros tipos de valoraciones.

\section{VALORES EPISTÉMICOS}

Larry Laudan (1941- ), Hilary Putnam y otros investigadores filosóficos propusieron el término de valores epistémicos para ese tipo de valores propios de la ciencia. El distinguir valores en el trabajo científico es un hecho muy común en la actualidad pero impensable por ejemplo hace cien años (Figura 1$)^{8}$.

Figura 1. Evaluación axiológica de valores epistémicos en un proyecto de investigación.

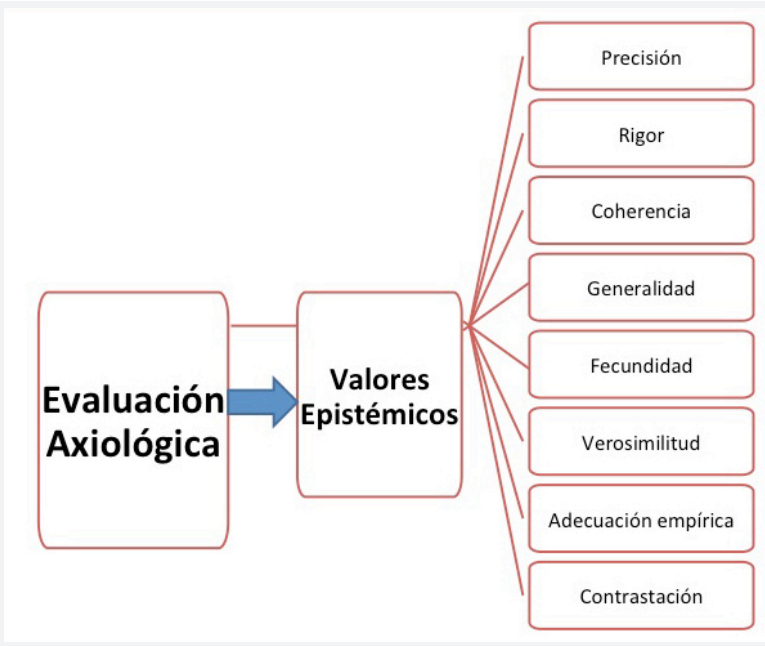

La parte de la filosofía que estudia los valores se denomina axiología o estimativa. A los valores se le pueden atribuir las siguientes características: el hecho intrínseco de «valer», la objetividad, la no independencia, la polaridad, la cualidad y la jerarquía?.

La noción de valor se amplió considerablemente a lo largo del siglo XX. Cuando se evalúa una Universidad o un Instituto de Investigación se está haciendo un juicio de valor institucional. Con la emergencia de los programas I+D a partir de la Segunda Guerra Mundial se configuraron unos nuevos valores y unos nuevos objetivos.

A esta transformación se le ha denominado de varias formas: modo 2 de conocimiento, ciencia postnormal y ciencia postacadémica $^{10}$. Echeverría prefiere el término de tecnociencia ${ }^{1}$. El investigador vasco acentúa el intenso componente tecnológico que posee en la actualidad la investigación científica, así como la necesaria cooperación entre científicos, ingenieros y técnicos. En investigación bíosanitaria también lo podríamos hacer extensivo a los estadísticos, epidemiólogos y a los documentalistas.

\section{EVALUACIÓN DE INNOVACIONES}

Entendiendo la investigación científica como una innovación, hemos de referir que son muchos los autores que vinculan la innovación a la creación de valor, entendiendo por tal la creación de valor económico ${ }^{11}$. En el caso de 
Schumpeter ${ }^{12}$, no sólo se habla de creación, sino también de destrucción de valor, conforme a su tesis de la «destrucción creativa». Aceptando que existiera un equilibrio en el mercado, una innovación auténtica siempre rompe ese equilibrio, porque provoca un cambio importante de los valores relativos, con las consiguientes ganancias para unos, y pérdidas para otros.

En este sentido la figura de Vannevar Bush ${ }^{13}$ también es capital por su aportación a las políticas de evaluación de la ciencia en su trabajo «Science, the endless frontier» (Ciencia, la frontera $\sin$ fin). Sus aportaciones no sólo fueron importantes en los Estados Unidos de América, sino que también recibió la Orden de Caballero del Imperio Británico y la Legión de Honor Francesa.

Trátese de innovaciones científicas, económicas, sociales, políticas o de otro tipo, uno de los requisitos para que algo nuevo sea innovador es que sea valorado positivamente por múltiples estamentos, en función de sus respectivos criterios. No hay innovación sin valoración previa.

\section{LOS COMITÉS DE ÉTICA DE LA INVESTIGACIÓN}

Los Comités de Ética de la Investigación (CEI) o los Comités Éticos de Investigación Clínica (CEIC) en España son organismos independientes, constituidos por profesionales sanitarios y no sanitarios, cuyo papel, tal como se define en las Normas de Buena Práctica Clínica ${ }^{14}$ de la Conferencia Internacional de Armonización, es «velar por la protección de los derechos, la seguridad y el bienestar de los sujetos que participan en un ensayo clínico o en un proyecto de investigación no experimental, y proporcionar una garantía pública al respecto mediante entre otras, la evaluación y emisión de un dictamen referente al protocolo del ensayo, la idoneidad del investigador, la adecuación de las instalaciones, así como a los métodos y materiales que serán utilizados para obtener y documentar el consentimiento informado de los sujetos del proyecto». Para nosotros la característica fundamental, diríamos que idiosincrásica de los CEIs, tiene que ser la independencia. Está claro también que ha de respetarse el principio de jerarquía dentro del comité.

Así pues el CEI garantiza que se observen las principales recomendaciones éticas aceptadas internacionalmente que deben regir la investigación biomédica: la Declaración de Helsinki, el Convenio de Oviedo y las recomendaciones del Consejo de Organizaciones Internacionales de Ciencias Médicas (CIOMS). La protección y el bienestar de los pacientes se fundamenta en los principios éticos básicos a contemplar en la ejecución de investigaciones que incluyen sujetos (establecidos en 1978 en el Informe Belmont) de: respeto a las personas (o principio de autonomía), benefi- cencia, justicia y (matizados en 1979 añadiendo un cuarto), la no maleficiencia ${ }^{13}$.

Así como el principio del respeto a las personas encuentra su máxima expresión en la obligatoriedad de obtener el consentimiento informado de los sujetos ${ }^{15} \mathrm{y}$ el principio de beneficencia en la necesidad de una evaluación de riesgos y beneficios realizada antes del inicio del estudio, el principio de justicia se preserva con una adecuada selección de los sujetos de la investigación y se considera maleficente la realización de un proyecto que no tenga validez científica, ya sea porque la hipótesis o el diseño no sean justificables, o porque no sea metodológicamente correcto. Por todo ello el CEI debe realizar una evaluación metodológica del protocolo (un proyecto de investigación mal diseñado no es éticamente aceptable) y una evaluación crítica de la hoja de información al paciente. $\mathrm{Y}$ todo ello extensivo no sólo a los diseños experimentales sino también a los observacionales, a la investigación cualitativa ${ }^{16}$ y a los diseños mixtos ${ }^{17}$. Esta evaluación metodológica no está reñida ni con la agilidad de funcionamiento del CEI ni con la normativa vigente.

En interesante señalar que en la bibliografía se reconocen tres tipos de CEIs según su funcionamiento ${ }^{18}$ : el CEI dictador, el CEI sumiso y el CEI conciliador. En el caso del dictador, prevalece su opinión, independientemente de lo opinado por el resto de los CEIC participantes; en el caso del sumiso, tiene en cuenta todas las aportaciones realizadas por los distintos CEIC implicados, aun cuando en ocasiones las peticiones fueran contradictorias. Por último, el conciliador tiene en cuenta todas las opiniones, pero traslada al promotor un resumen de la evaluación de todos ${ }^{19}$. Altisent y cols. ${ }^{20}$ han defendido una cualificación mínima para la pertenencia a un comité de ética clínica, quizás esos requisitos deban de ser aún mayores para los integrantes futuros de los $\mathrm{CEIs}^{21}$.

\section{INTERÉS PARA EL FARMACÉUTICO}

El interés para el farmacéutico sería clasificable dentro de los principios del Uso Racional del Medicamento (URM). Estos principios son la eficacia, la seguridad, la adecuación y el coste. Estos cuatro principios se pueden relacionar con los siguientes valores éticos, el principio de eficacia con el de beneficiencia, el principio de seguridad con el de no maleficiencia, el de adecuación con el respeto a la autonomía del paciente y el de coste con el principio de justicia social. Los principios de URM presiden el trabajo diario del farmacéutico.

\section{PROTECCIÓN DE IDEAS Y DIFUSIÓN DE LA INVES- TIGACIÓN}

Entendemos que otra función importante de los CEIs es la protección del conocimiento real o potencial que se deriven 
del desarrollo de un proyecto de investigación ${ }^{22}$. Existen al menos cuatro razones para proteger los resultados de la investigación: 1) avanzar en el desarrollo de las ideas aplicables, 2) conseguir que las ideas se apliquen produciendo el beneficio social que se pretendía,3) obtener un retorno de la inversión realizada para poder seguir investigando de forma independiente, y con ello llegar a la creación de empleo y al crecimiento social y 4) evitar que otros se beneficien de la explotación de invenciones propias sin nuestro permiso.

Judit Pich y colaboradores informaron hace años ${ }^{23}$ que tan sólo el $27 \%$ de los ensayos clínicos comenzados se presentaban en eventos científicos y que sólo el 31\% llegaban a ser publicados en revistas con sistema de revisión por pares. Parece lógico que una tarea complementaria de los CEI sea también velar por el proceso de edición y difusión de los ensayos clínicos que revisan ${ }^{24}$. Parece ser que la crisis eco- nómica no ha hecho disminuir la carga de trabajo en los $\mathrm{CEIs}^{25}$

\section{DISCUSIÓN}

Desde el Royalist Compromise y el Catecismo Positivista (siglos XVII-XIX; punto de partida no axiológico) la investigación científica ha caminado hacia un tiempo histórico de apreciación de valores epistémicos (siglo XX; actual situación axiológica). Los trabajos de Merton ${ }^{1} \mathrm{y}$ de Putnam ${ }^{5}$ han sido fundamentales en este sentido. En ese escenario, nosotros comprendemos la existencia de los CEIs en el panorama sanitario español sobre todo dentro de la evaluación de las innovaciones. Nosotros también aceptamos el aforismo de que «Lo que nos es metodológicamente adecuado, no es éticamente correcto» por el principio de la no maleficencia y por la situación axiológica simultánea de la investigación científica ${ }^{1}$.

Figura 2. Evolución de las funciones clásicas a las axiológicas de los Comités de Ética de la Investigación.

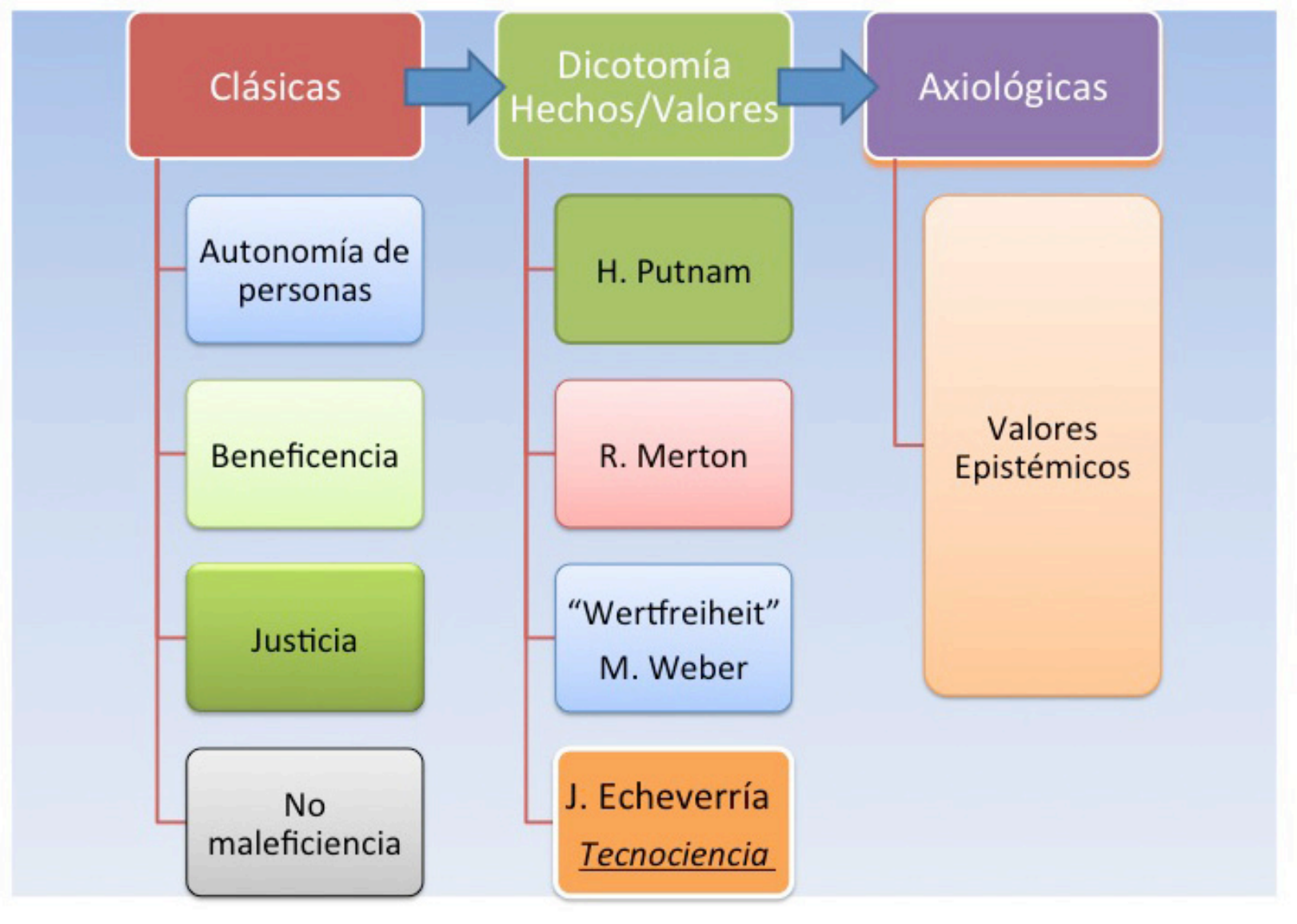

Tan es así, que finalmente patrocinamos ${ }^{1,8,11,26}$ dos tesis en este estudio (Figura 2). La primera afirma que la existencia actual de los CEIs es un hecho congruente con el desarrollo histórico reciente de la epistemología ${ }^{27}$ y la segunda defiende que además de las ya descritas, una de las funciones más importantes de los CEIs es la evaluación axiológica (valores epistémicos ${ }^{29}$ ) de los proyectos de investigación que a ellos se presenten ${ }^{30}$. 


\section{ADDENDA}

Los autores agradecen la labor crítica del revisor/a de Ars Pharmaceutica porque ha enriquecido notablemente este manuscrito.

Un resumen argumental muy resumido de este trabajo fue presentado como póster en el XIX Congreso de la Sociedad Andaluza de Calidad Asistencial celebrado en Sevilla en Noviembre de 2014. https://www.researchgate.net/publication/271013520_La_Evaluacin_Axiolgica_de_los_Proyectos_en_los_Comits_de_tica_de_la_Investigacin

\section{BIBLIOGRAFÍA}

1. Echevarria-Ezponda J: Los valores de las ciencias. Investigación y Ciencia (ed. española). 2014; 452: 44-45.

2. Proctor, R N : Value-free science ? Purity and Power in Modern Knowledge. University Press. 1991. Harvard College. p.7. Disponible en : http://books.google.es/books?id=yol8zpXx 3sYC\&pg=PA7\&lpg=PA7\&dq=\%22royalist + compromise $\% 22$ + royal + society $\&$ source $=$ bl\&ots $=6$ XDSaueb80\&sig $=\mathrm{dmSyahB}$ GOcuJ8QaBJ2H4UcI1g3c\&hl=es\&sa=X\&ei=YIDKU_XRAaO h0QXdoIHAAg\&ved $=0 C C g Q 6 A E w A Q \# v=$ onepage $\& q=\% 22$ royalist $\% 20$ compromise $\% 22 \% 20$ royal $\% 20$ society \&f=false

3. Artigas, M: Lógica y ética en Karl Popper. EUNSA. Pamplona. 1998.

4. Defez i Martín, A : Pensamiento y lenguaje en el primer Wittgenstein. AGORA. Papeles de Filosofía. 2000; 19/1 : 153-167. Disponible en : https://dspace.usc.es/bitstream/10347/1156/1/pg_155-170_agora19-1.pdf

5. Putnam, H: The collapse of the fact/value dichotomy and other essays. Cambridge, MA: Harvard University Press. 2002.

6. http://es.wikipedia.org/wiki/Ethos. Visitado el 31 de Diciembre de 2014.

7. Ziman, J: Real Science: what it is, and what it means. Cambridge: Cambridge University Press. 2000.

8. Echeverría , J : La Revolución Tecnocientífica. CONfines. 2005 ; 1/2 : 9- 15. Disponible en : http://confines.mty.itesm.mx/ articulos2/EcheverriaJ.pdf?origin=publication_detail

9. Ferrater Mora, J: Diccionario de Filosofía Abreviado. Edhasa. Barcelona. 2008; p: 364-366.

10. Jiménez-Buedo , $\mathrm{M}^{\mathrm{a}}$, Ramos-Vielba, I: ¿Más allá de la ciencia académica?: modo 2, ciencia posnormal y ciencia posacadémica. Arbor. 2009; 185: 721-737. Disponible en: http://arbor. revistas.csic.es/index.php/arbor/article/viewArticle/326

11. Echevarria-Ezponda J: Evaluar las innovaciones y su difusión social. ISEGORÍA. 2013; 48:173-184. Disponible en : http:// isegoria.revistas.csic.es/index.php/isegoria/article/viewArticle/816

12. Schumpeter, J: Business Cycles, New York, McGraw Hill. 1939.
13. Pielke, R Jr : In Retrospect : Science - The endless frontier. Nature 2010 : 922-923. Accesible en : http:/ / www.nature.com/ nature/journal/v466/n7309/full/466922a.html

14. Riba, N , Peñataro, JS : La labor de los comités de ética de investigación en los ensayos clínicos. Lo que es y lo que debería ser. En : Dal-Ré, R, Carné, X, y Gracia, D: Luces y sombras en la investigación clínica. Ed. Triacastela. 2013. p.114-126.

15. Simón P: Diez mitos en torno al consentimiento informado. An. Sist. Sanit. Navar. 2006; 29 : 29-40. Disponible en : http:/ / scielo.isciii.es/pdf/asisna/v29s3/original3.pdf

16. Barrio-Cantalejo, M, Simón-Lorda, P : Problemas éticos de la investigación cualitativa. Med. Clin (Barc.). 2006; 126 : 418423.

17. González-García L, Gómez-González C, Chemello C, Cubilesde la Vega Ma D, Santos-Lozano J M, Ortega-Calvo M : Triangulación de un estudio cualitativo mediante regresión logística. Index Enferm . 2014; 23: 80-84. Disponible en: http:// dx.doi.org/10.4321/S1132-12962014000100017.

18. Gálvez Múgica MA, Pablo López de Abechuco I : El proceso de evaluación de un ensayo clínico desde la perspectiva de un comité ético de investigación clínica. Rev Clin Esp. 2007; $207: 29-33$.

19. Redondo-Capafons S, Salort-Llorca C, Pla-Poblador R, Quintana S: Ensayos clínicos. Valoración del tipo de aclaraciones solicitadas y homogeneidad en la revisión por los comités éticos de investigación clínica. Med Clin (Barc). 2009; 133: $23-5$.

20. Altisent R, Delgado-Marroquín MT, Martín-Espildora N : Toward a model that encourages the recruitment of ethics consultants with clinical experience. Am J Bioeth. 2014; 14: 28-30.

21. Baños JE, Lucena MI, Serés E, Bosch F: Reflections on running training workshops for research ethics committee members in Spain between 2001 and 2008. Croat Med J. 2010 ;51:5529. Disponible en: http://www.cmj.hr/2010/51/6/21162168. htm

22. Musoles, S: Protección de las ideas y de los resultados de investigación. Med Clin (Barc). 2012;139:688-693.

23. Pich J, Carné X, Arnaiz JA, Gómez B, Trilla A, Rodés J : Role of a research ethics committee in follow-up and publication of results. Lancet. 2003 ; $361: 1015-6$.

24. Schroter S, Plowman R, Hutchings A, Gonzalez A: Reporting ethics committee approval and patient consent by study design in five general medical journals. J Med Ethics. $2006 ; 32$ :718-23. Disponible en : http:/ / www.ncbi.nlm.nih.gov/pmc/ articles/PMC2563342/

25. Redondo Capafons S, Arcenillas P, Giménez N, March López P, Soriano L, Pla R, Quintana S : Impacto de la crisis económica en la actividad de un comité ético de investigación clínica. Farm Hosp. 2014 ; 38 : 454-60. Disponible en : http://www. grupoaulamedica.com/fh/pdf/7522.pdf 
26. Echevarría, J : Tecnociencia, tecnoética y tecnoaxiología. Revista Colombiana de Bioética. 2010; 5 : 142 - 152. Disponible en : http://www.bioeticaunbosque.edu.co/publicaciones/ Revista/pdf_revistacolbio/revcolbio5_1.pdf\#page=143

27. Bosch, F, Baños, JE: Tendiendo puentes: la utilidad de la historia de la ciencia para comprender el proceso de investigación y desarrollo de medicamentos. Educ Med. 2010; 13: 255-262.

28. Allen, PM , Varga , L : Complexity: The Co-evolution of Epistemology,
29. Axiology and Ontology. Nonlinear Dynamics, Psychology, and Life Science. 2007, 11. 19-50. Disponible en : Ortega Calvo M, Román Torres P, Lapetra Peralta J: La epistemología como propedéutica de la investigación sanitaria. Gac Sanit.2011; 25:79-83. Disponible en : http://scielo.isciii.es/pdf/gs/ v25n1/especial.pdf

30. Balazs , Z : The instantation of values. SpringerPlus 2013, 2:166. Disponible en : http://link.springer.com/article/10.1186/2193-1801-2-166/fulltext.html 